\title{
Common Bile Duct Obstruction due to Ascariasis
}

\author{
Sabrina Bhattacharya ${ }^{1}$, Priyal Taribagil ${ }^{2}$, Sanjay Taribagil ${ }^{3}$ and Vish Bhattacharya ${ }^{3 *}$ \\ ${ }^{1}$ Foundation Doctor, Royal Victoria Infirmary, Newcastle upon Tyne NHS Foundation Trust, UK \\ ${ }^{2}$ Academic Foundation Doctor, Barnet Hospital, Royal Free London NHS Foundation Trust, UK \\ ${ }^{3}$ Consultant General and Upper Gastrointestinal Surgeon, Queen Elizabeth Hospital NHS Foundation Trust, UK
}

*Corresponding author: Vish Bhattacharya, Consultant General and Upper Gastrointestinal Surgeon, Queen Elizabeth Hospital

NHS Foundation Trust, Gateshead, NE9 6SX, UK

\section{ARTICLE INFO}

Received: May 28, 2020

Published: 幽 June 10, 2020

Citation: Sabrina B, Priyal T, Sanjay T, Vish B. Common Bile Duct Obstruction due to Ascariasis. Biomed J Sci \& Tech Res 28(2)2020. BJSTR. MS.ID.004616.
ABSTRACT

Ascaris Lumbricoides (also known as roundworm), affects approximately one billion individuals across the globe and is considered the most prevalent helminthic infection. Although asymptomatic in majority of the population, these nematodes have the potential to present with intestinal obstruction and perforation. Biliary ascariasis leading to Common Bile Duct (CBD) obstruction is an uncommon manifestation. Abdominal ultrasound is the non-invasive investigation of choice with a high degree of sensitivity and specificity. Pyogenic cholangitis, liver abscess formation and pancreatitis are potentially life-threatening complications of untreated Hepatobiliary Ascariasis (HBA) hence early diagnosis and appropriate intervention is essential. Surgical intervention in combination with appropriate anthelmintic medications is often required to treat complex HBA. We present an interesting case of complex HBA and review of literature.

\section{Background}

Ascaris Lumbricoides (also known as roundworm), affects approximately one billion individuals across the globe and is considered the most prevalent helminthic infection. This infection predominates in areas of Sub-Saharan Africa, South Asia, China and Latin America [1]. Common clinical features of this helminth infection are small bowel obstruction, upper gastrointestinal bleed and malabsorption [2]. In terms of biliary disease, ascariasis can manifest as biliary colic, pancreatitis, strictures of the bile duct and liver abscesses [3]. Obstructive jaundice due to the presence of roundworms in the common bile duct lumen is a very rare presentation which will be discussed in the case report. The infective life cycle of Ascaris Lumbricoides begins with the presence of nematode eggs in faeces- contaminated soil. In warm, moist conditions the eggs mature in faeces contaminated soil for 2-8 weeks and remain viable for a duration of 17 months. This disease is transmitted via the faecal-oral route and humans are generally infected by ingestion of food, raw vegetable or water contaminated with mature ova (1). Once ingested, the larvae are released in the duodenum after being stimulated by gastric juices and the resultant rhabditiform larvae migrate into the caecum. Here they infiltrate the epithelium, entering the portal vein and hepatobiliary system (2). In some cases, they can spread via the hepatic veins and lymphatic system to reach the lungs (1). They then pass through the capillary wall into the alveolar spaces. They ascend the larynx and hypopharynx before being swallowed. They undergo a process of maturation throughout this journey resulting in adult worms that reside in the jejunum. These adult female worms produce a large number of eggs that pass through the faeces to complete the cycle.

\section{Case Report}

52-year-old generally fit and well rural farmer in Dervan, a small Indian village situated in Maharashtra state, presented to the annual surgical camp with jaundice, weight loss and anorexia. Abdominal examination revealed right upper quadrant tenderness. Pale stools and dark urine were noted. Investigations included abdominal ultrasound and intraoperative cholangiogram that confirmed common bile duct obstruction due to gallstones and ascariasis. Patient underwent open common bile duct exploration with removal of worms and pigmented stones (Figures 1-3). 
Cholecystectomy was subsequently carried out. Numerous pigmented stones were extracted in conjunction with multiple dead
Ascaris worms. He received a full course of oral Mebendazole and was discharged after an uneventful recovery.

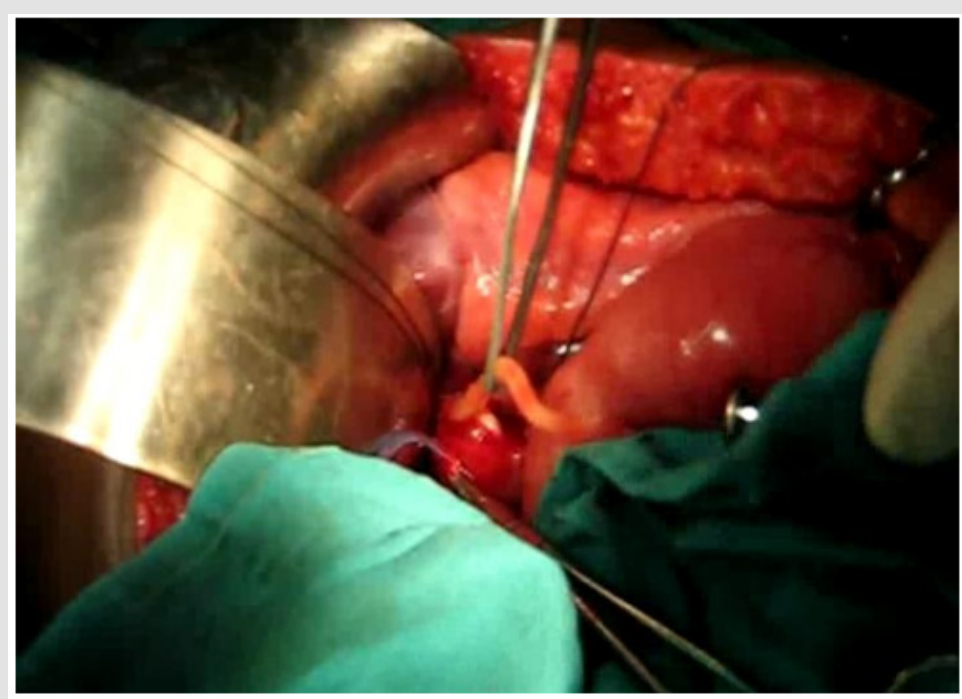

Figure 1: Open exploration of common bile duct and removal of worms.

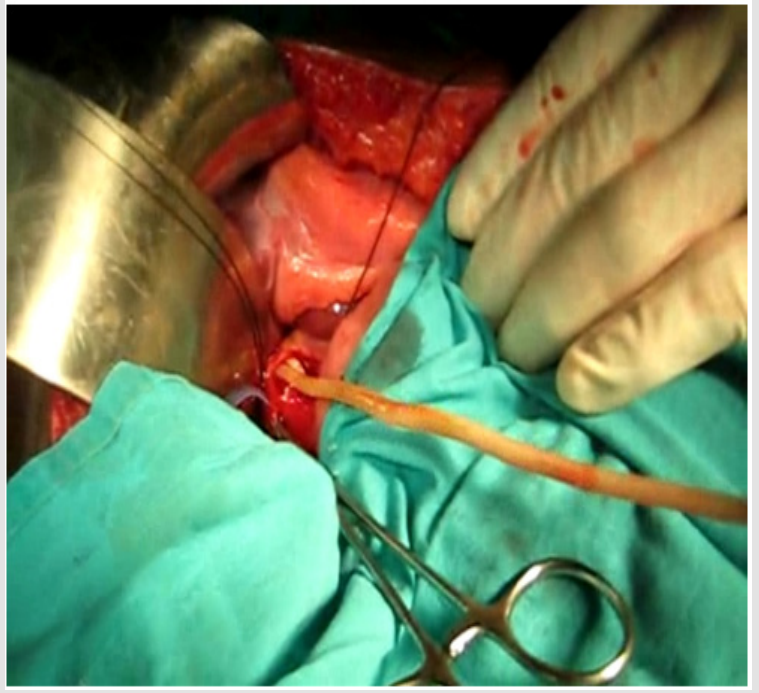

Figure 2: Open exploration of common bile duct and complete removal of worms.
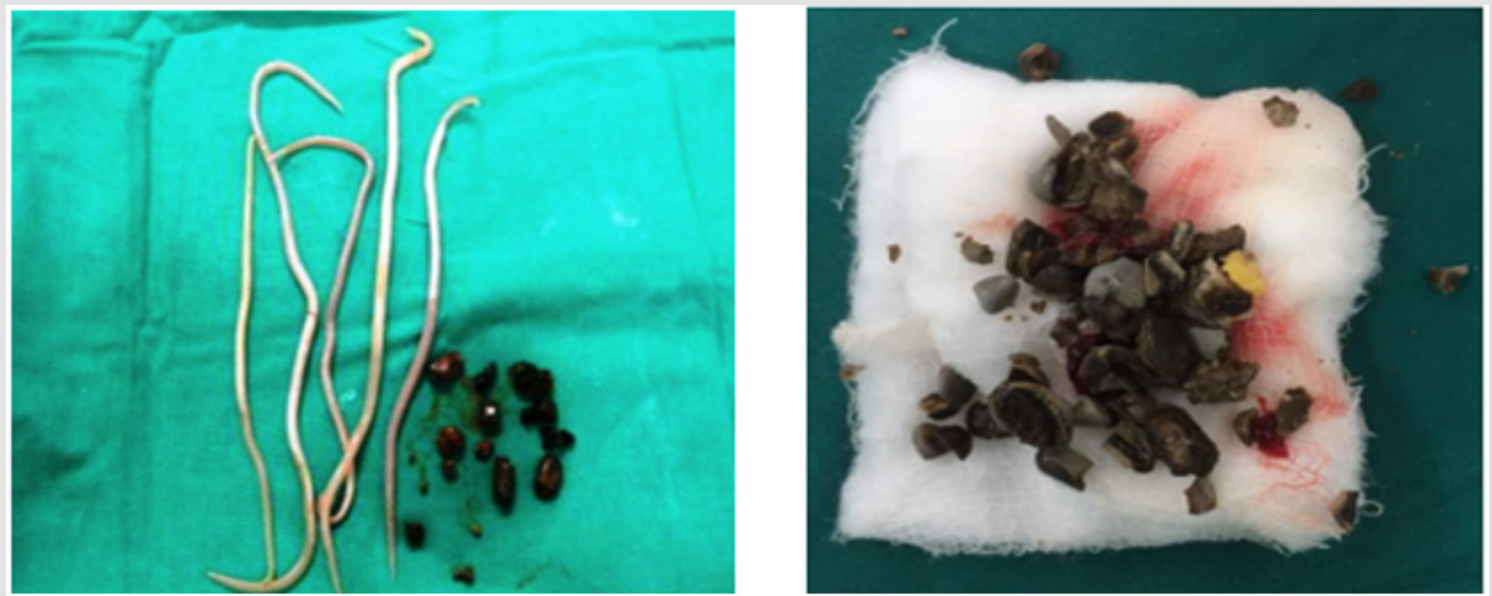

Figure 3: Removal of five dead Ascaris worms and multiple pigment stones completed. 


\section{Discussion}

Ascaris lumbricoides, the largest common nematode causing human ascariasis, infects over one billion of the world's population $[4,5]$. Although it is a worldwide disease mainly due to increases in international travel, the major burden is felt by the tropical countries which have moist soil and good rain. In the tropics, up to $70 \%$ of the children are found to be infected [6]. South-East Asian countries and China show variable prevalence rates of 41$92 \%$ while in parts of Africa, it is estimated to be higher at $95 \%$ [7]. In India, the highest rates of infection with this helminth is found in Tamil Nadu (85\%) and Kashmir (90\%) [5,7]. The small intestine is the most frequent site for infection however migration and infiltration of the helminth into the biliary tract can lead to a number of acute and chronic complications. Biliary ascariasis is a rare cause of obstructive jaundice. Risk factors for acquiring this helminth infection include poor hygiene, pollution involving untreated sewage (often implicated in farming) and malnutrition. These factors partially account for increased infection rates in tropical countries as mentioned above [4].

In the majority of cases, intestinal infection is asymptomatic or leads to mild symptoms including abdominal pain, weight loss or change in bowel habit. Commonest clinical manifestations of biliary ascariasis include biliary colic, acute cholecystitis, acute cholangitis, pancreatitis and hepatic abscess formation [8-10]. More severe clinical disease is associated with a heavy worm load (13-40 worms) accounting for 10,000-20,000 deaths per year across the globe [5]. Heavy worm infestation is considered a pre-requisite for the organism to reach the duodenum from the natural habitat of the jejunum. A host reaction can alter the secretory responses of the gut and therefore intestinal tone. The worm enters the biliary system by entering the ampulla of Vater. It can often enter the cystic duct and common bile duct but has rarely been reported to enter the gallbladder itself [5]. Predisposing factors for CBD infiltration include a prior history of cholecystectomy [3]. This is thought to be due to the effect of iatrogenic CBD dilation and cholecystokinin release which causes relaxation of the Sphincter of Oddi. These factors promote easy migration of the helminth into the biliary tree [3].

\section{Diagnosis}

Diagnosis is best achieved by ultrasonography [11]. The high sensitivity and specificity enable accurate visualisation of the worm in the biliary tree as well as any positional changes over time. Typical ultrasound findings include the presence of a hyper echogenic tubular structure without acoustic shadowing. Endoscopic ultrasound or real time - percutaneous ultrasound has been used to demonstrate the movement of the worm [10]. It has been noted however that ultrasonography may not be able to identify hepatobiliary ascariasis when worms are present in the duodenum or ampullary orifice [11]. Endoscopic Retrograde
Cholangiopancreatography (ERCP) is helpful in these situations both for diagnostic and therapeutic aspects. The worms appear commonly as linear, smooth filling defects without distal acoustic shadowing. They have also been reported as forming parallel filling defects, also known as the "railway tract" sign, curved defects or transverse loops spanning across the duct. Where worms have entered the gallbladder, they are more easily visualised on Computed Tomography (CT) imaging and appear as coiled tubular structures [12-15]. Blood tests can be useful in detecting an inflammatory response however antibodies are seldom of use as there can be significant cross-reactivity from infection with other helminthic organisms [5]. A full blood count may show eosinophilia in cases presenting with HBA. Liver function tests (bilirubin, transaminases, gamma GT and alkaline phosphatase) may also be elevated depending on the degree of biliary obstruction. Stool examination is the only specific test however this may be often negative.

\section{Relation with Bile Duct Stones}

In tropical areas, the prevalence of gallstones is significantly lower due to high dietary fibre and low cholesterol diet. Acalculous cholecystitis is a more common clinical presentation. However, there is speculation that hepatobiliary ascariasis may be associated with biliary stone formation. The presence of existing biliary sludge is thought to provide a nidus for adult worms and ova to initiate calculus formation. This has been supported by histological evidence of Ascaris remnants and eggs found within the biliary stones [14]. Bacterial infections, secondary to the biliary obstruction further precipitate this process. Interestingly where patients have been found to have HBA, stones were seldom found in the gallbladder. Majority were identified in the intrahepatic biliary ducts. As noted previously this may be because the worm does not tend to enter the gallbladder itself [5].

\section{Recurrent Pyogenic Cholangitis}

Pyogenic cholangitis as a result of ascariasis was first noted in the Far East where it was referred to as 'Asiatic or Oriental cholangiohepatitis' [15]. Features of recurrent pyogenic cholangitis include presence of intrahepatic bile duct stones and chronic bacterial infection secondary to HBA. Patients affected by this are generally malnourished and have a low Body Mass Index (BMI). They characteristically present with right upper quadrant pain, rigors and cholestatic jaundice [5]. Imaging shows the presence of sludge in the intra and extrahepatic biliary systems. Where biliary ducts have been frequently affected, recurrent pyogenic cholangitis can present without active ascariasis. This can lead to scar formation and hepatic abscesses. Recurrent biliary infection can result in dysfunction of the sphincter of Oddi from repeated insult by the worm [5]. It has been reported that 5\% of those affected with HBA will develop recurrent pyogenic cholangitis at 2 years [5]. Stones are more commonly a feature in recurrent pyogenic cholangitis 
and are formed by part of the ascaris worm [16]. These long-term sequelae are important to consider when deciding on management for patients with ascariasis.

\section{Management}

A variety of oral anthelmintic medications are available for use. These act to paralyze the worm in the adult phase however do not work in the larval stage of the lifecycle. Pyrantel pamoate works by spastic paralysis of the worm and is the most commonly used; it usually leads to worm clearance by 3 days. Other medications used include piperazine citrate which causes flaccid paralysis of the worm [16]. The dead worm remnants trigger a large inflammatory reaction which has the potential to cause fibrosis and strictures. To monitor efficacy of treatment, eosinophil count can be checked, and persistent eosinophilia correlates with treatment failure. Indications of surgical intervention include: failure of medical management - worms have not been expelled from the biliary system after 3 days, pancreatitis, ascending cholangitis, obstructive jaundice and sepsis . Other considerations for urgent operative management include biliary strictures or worms within the gallbladder itself [16]. Where worms have infiltrated the gallbladder, cholecystectomy is the treatment of choice. ERCP can be used for acute pyogenic cholangitis and biliary colic. This is generally successful in $90 \%$ of cases [16]. Complications of ERCP are the same as when done for any other indication (pancreatitis, haemorrhage, cholangitis and perforation). Success of endoscopic extraction makes it the treatment of choice in HBA [16]. Presence of multiple calculi or a large worm, warrants an open approach to relieving the biliary obstruction. Of note, as many of these cases occur in tropical areas and often rural locations without endoscopic facilities, open surgery may be the first line of treatment as in our case.

\section{Summary}

HBA should be considered as a main differential in patients presenting with vague abdominal pain with or without specific biliary signs such as jaundice in endemic areas. Ultrasonography or CT scanning remains the best diagnostic tool for this presentation. Generally, HBA carries a good prognosis and most often respond to conservative therapy with oral anthelminthic medications. In non-responders or when patients are acutely unwell, endoscopic or surgical removal of the worm is necessary. Associated intestinal obstruction and acute pancreatitis should be looked for especially in children. Long-term effects of HBA include recurrent pyogenic cholangitis, liver abscesses formation and pancreatitis, therefore a high index of suspicion and early intervention should be considered.

\section{References}

1. Cook GC (1989) Ascariasis and its prevention and control. Trans R Soc Trop Med Hyg 83(4): 538.

2. Corvino DF de L, Horrall S (2020) Ascariasis. StatPearls.

3. (2019) Prevention C-C for DC and CDC - Ascariasis - Biology.

4. LS G, D I, SD D (2018) Prevalence and Intensity of Ascaris Lumbricoides Infections in Relation to Undernutrition Among Children in a Tea Plantation Community, Sri Lanka: A Cross-Sectional Study. BMC Pediatr 18(1).

5. Das AK (2014) Hepatic and Biliary Ascariasis. J Glob Infect Dis 6(2): 6572.

6. Louw JH (1966) Abdominal complications of Ascaris lumbricoides infestation in children. Br J Surg 53(6): 510-521.

7. Thein Hlaing, Myat Lay Kyin, Hlaing Mya, Maung Maung (1990) Role of ascariasis in surgical abdominal emergencies in the Rangoon Children's Hospital, Burma. Ann Trop Paediatr 10(1): 53-60.

8. David WT, Nesheim MC, Pawłowski ZS (1985) Ascariasis and its public health significance: a volume based on the agenda and discussions of the 1984 Banff conference, organized by WHO Parasitic Diseases Programme and Division of Nutritional Sciences, Cornell University, New. Taylor \& Francis 289.

9. Alam S, Mustafa G, Rahman S, Kabir SA, Rashid HO, et al. (2010) Comparative Study on Presentation of Biliary Ascariasis with Dead and Living Worms. Saudi J Gastroenterol 16(3): 203-206.

10. Jethwani U, Singh GJ, Sarangi P, Kandwal V (2012) Laproscopic Management of Wandering Biliary Ascariasis. Case Rep Surg : 1-4.

11.S D, K T (1995) Biliary Ascariasis: Sonographic Findings. AJR Am J Roentgenol 164(3): 767-768.

12. Wasadikar PP, Kulkarni AB (1997) Intestinal obstruction due to ascariasis. Br J Surg 84(3): 410-412.

13. Maddern GJ, Dennison AR, Blumgart LH (1992) Fatal ascaris pancreatitis: an uncommon problem in the west. Gut 33(3): 402-403.

14. ES Palmer, Maurice M (2020) Reeder The Imaging of Tropical Diseases: With Epidemiological, Pathological and ... Philip.

15. Verweij KE, Van Buuren H (2016) Oriental cholangiohepatitis (recurrent pyogenic cholangitis): a case series from the Netherlands and brief review of the literature. The Netherlands journal of Medicine 79(9): 401-405.

16. IJ B, SN C, JE K, PC B, J T (1998) Management of Hepatobiliary and 
ISSN: 2574-1241

DOI: 10.26717/BJSTR.2020.28.004616

Vish Bhattacharya. Biomed J Sci \& Tech Res

(C) (i) This work is licensed under Creative

Submission Link: https://biomedres.us/submit-manuscript.php

\begin{tabular}{ll} 
BIOMEDICAL & Assets of Publishing with us \\
RESEARCHES & - Global archiving of articles \\
& - Immediate, unrestricted online access \\
\hline
\end{tabular}

